\title{
Environmental issues facing chemical, biological, radiological, and nuclear risks
}

\author{
Marie-Thérèse Menager ${ }^{1}$ - Eric Ansoborlo ${ }^{2}$ - David Pignol ${ }^{3,4,5}$
}

Received: 24 November 2015 / Accepted: 27 November 2015 /Published online: 15 December 2015

(C) Springer-Verlag Berlin Heidelberg 2015

This special issue of Environmental Science and Pollution Research highlights selected papers presented at the first international conference entitled "CBRN, research and innovation" (CBRN-RI in the following) which was held on March 16-18, 2015 at Antibes-Juan-les-Pins in France. This conference was co-organized by the French Alternative Energies and Atomic Energy Commission (CEA), the Armament procurement Agency (DGA), the Army Health Services, the Alpes-Maritimes Fire and Rescue Services (SDIS06), and Nice Conference Association.

This special issue covers the topics of detection, remediation, and prevention of environments exposed to highly hazardous Chemical Biological Radiological and Nuclear (CBRN) agents following conflicts, natural catastrophes, industrial incidents, or terrorist acts. Worldwide, research, and industrial capacities have been undertaken to provide adapted answers to these new challenges. Besides existing conferences which give the opportunity for industrials to present their newly developed equipment, the aim of the CBRN-RI conference was to create a specific and unique communication space

Responsible editor: Philippe Garrigues

David Pignol

david.pignol@cea.fr

1 Commissariat à l'Energie Atomique et aux Energies Alternatives, Direction des Sciences du Vivant, BP 6

92265 Fontenay-aux-Roses, France

2 Commissariat à l'Energie Atomique et aux Energies Alternatives, DEN/DRCP/CETAMA, Marcoule, F-30207 Bagnols-sur-Cèze, France

3 CEA, DSV, IBEB, Lab Bioenerget Cellulaire, Saint-Paul-lez-Durance F-13108, France

4 CNRS, UMR Biol Veget \& Microbiol Environ, Saint-Paul-lez-Durance F-13108, France

5 Aix-Marseille Université, Saint-Paul-lez-Durance F-13108, France dedicated to research and innovation. It emerged as one of the best platforms for academic exchange and synergy with industrials of the field and provided a forum for researchers to address recent research results and to discuss various technical issues in the following themes: (i) pollution detection, (ii) human and environmental decontamination, (iii) protection, and (iv) therapeutics.

Although investment of considerable financial, human, and scientific resources to develop medical countermeasures are developed to rapidly and effectively respond to a deliberate chemical, biological, radiological, or nuclear attack, tools for environment survey and protection are also now of growing interest in the field of NRBC-risks. This special issue contains articles that illustrate enzymatic decontamination processes with the case of organophosphates and environmental detection of toxic compounds as exemplified for toxic heavy metals. Studies focused on dedicated developments for efficient air sampling and detection of chemical warfare agents are also proposed. Original approaches used for the rapid identification of pathogenic bacteria or bacterial toxins in the field are also addressed.

This Special Issue cannot hope to encompass the diversity and the originality of the research presented at CBRN-RI 2015; however, as editors, we hope that the Research Articles collected here will encourage environmental scientists to consider participating in the second CBRN-RI edition which is to be held in Lyon (France) from March 20 to 23, 2017.

Finally, we sincerely thank all of the sponsors of the 2015 CBRN-RI conference. We also thank all the participants in this stimulating symposium, and in particular, the authors of the papers included in this Special Issue, which reflects the current international activities in the field. The guest editors of this Special Issue hope these papers are a valuable contribution to the scientific literature and useful to researchers and industrials worldwide involved in CBNR concerns. Dr. Philippe Garrigues, Editor-in-Chief of Environmental Science and Pollution Research, and his Editorial team are warmly thanked for their interest on this topic and handling of the review process. 


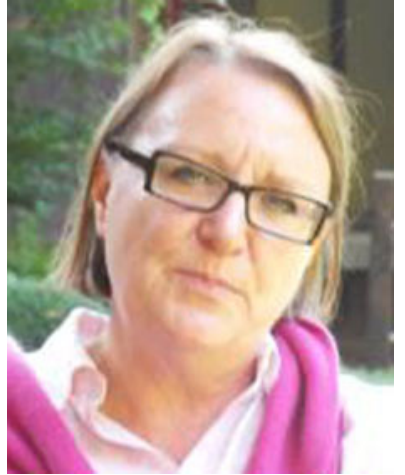

Dr. Marie-Thérèse Menager is a scientific manager of the health science division (DSV) of the Commissariat à 1'Energie Atomique et aux Energy alternatives (CEA). She is an expert in the field of geochemistry and environmental science. Since 2001, she has been in charge of animating and coordinating several multidisciplinary national programs led by the CEA in the fields of nuclear toxicology, technology for health, and biotechnology against CBRN-risks. In 2015, she was a leading member of both the scientific board and the organizing committee of the first international congress entitled "CBRN, research and innovation."

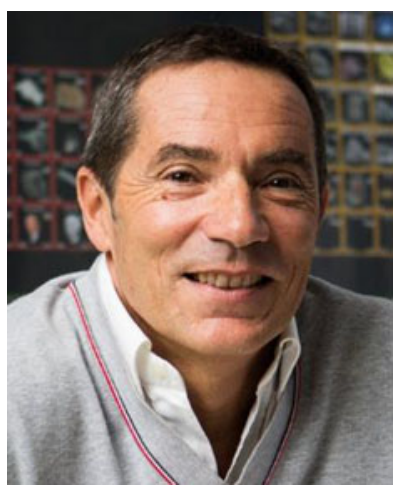

Dr. Eric Ansoborlo is an international expert at the Nuclear Energy Division of the French Alternative Energies and Atomic Energy Commission (CEA). He is also an expert in internal dosimetry for the International Commission on Radiological Protection (ICRP) and a representative for France at the United Nations Scientific Committee on the Effects of Atomic Radiations (UNSCEAR). His background and field of scientific expertise are radiochemistry, analytical chemistry, and speciation applied to life science and environment. He has been specifically involved during the last 15 years in a national Nuclear Toxicology programme of CEA devoted to radiotoxicology and focusing on biokinetics and metabolism of main radionuclides encountered in nuclear industry (e.g., tritium, cobalt, cesium, iodine, polonium, lanthanides, and actinides...) and also on the treatment in case of internal contamination also named decorporation.

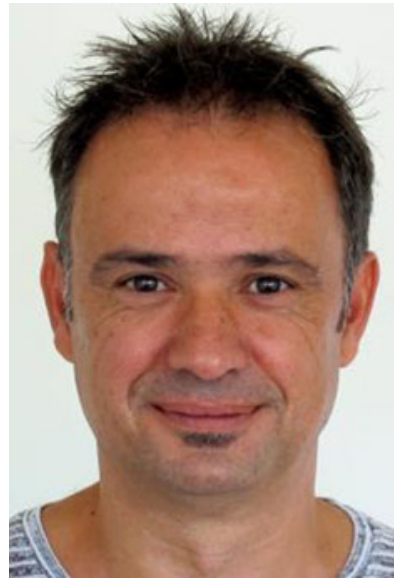

Dr. David Pignol is a research director at the health science division (DSV) of the Commissariat à l'Energie Atomique et aux Energy alternatives (CEA). He is the head of the Bioenergetics Laboratory (LBC), a molecular microbiology laboratory located in Cadarache (Institute of Environmental Biology and Biotechnology). His group has internationally renowned expertise in the characterization of mechanisms responsible for the adaptation of different bacterial species (photosynthetic, radiotolerant, and magnetotactic bacteria) to their environments (web site of the LBC: http://ibeb.cea.fr/dsv/ibeb/ english/Pages/laboratories/lbc.aspx). Since his Ph.D defense in 1994 (Institute of Structural Biology, Grenoble), he published over 75 peerreviewed papers in molecular microbiology, focussing on the mechanisms that govern bacterial resistance, acquisition, sequestration, and mineralisation of physiological and toxic heavy metals. Using multidisciplinary approaches, he tackled more recently with his group the field of metal biomineralization and was able to identify and characterize key proteins from magnetotactic bacteria, unique microorganisms able to produce intracellular magnetite nanomagnets, becoming one pioneering lab in the field. The fundamental studies are always developed in his laboratory keeping in mind the potential biotechnological applications (three active patents in the field of heavy metal depollution). 